\title{
Perception of Threatening Intention Modulates Brain Processes to Body Actions: Evidence From Event-Related Potentials
}

\author{
Guan Wang ${ }^{1 \dagger}$, Pei Wang ${ }^{2 *}$, Junlong Luo ${ }^{3}$ and Wenya Nan ${ }^{3}$ \\ ${ }^{1}$ School of Education Science, Huaiyin Normal University, Huaian, China, ${ }^{2}$ Department of Special Education, Faculty of \\ Education, East China Normal University, Shanghai, China, ${ }^{3}$ Department of Psychology, Shanghai Normal University, \\ Shanghai, China
}

Efficiently perceiving a threatening intention conveyed by others' bodily actions has great survival value. The current study examined if the human brain is sensitive to differences in intentions that are conveyed via bodily actions. For this purpose, a new intention categorization task was developed in which participants sat in front of a computer screen on which the pictures of highly threatening $(\mathrm{HT})$, moderately threatening $(\mathrm{MT})$, and

OPEN ACCESS

Edited by:

Kielan Yarrow,

City, University of London,

United Kingdom

Reviewed by:

Alejandro Galvez-Pol,

University College London,

United Kingdom

Beatriz Calvo-Merino,

City, University of London, United Kingdom

*Correspondence:

Pei Wang

wangg956892@126.com

† Joint first authors

Specialty section

This article was submitted to Perception Science, a section of the journal

Frontiers in Psychology

Received: 18 January 2018

Accepted: 19 October 2018

Published: 27 November 2018

Citation:

Wang G, Wang P, Luo J and

Nan W (2018) Perception

of Threatening Intention Modulates Brain Processes to Body Actions:

Evidence From Event-Related

Potentials. Front. Psychol. 9:2149.

doi: 10.3389/fpsyg.2018.02149 non-threatening (NT) body actions were presented randomly. Participants were asked to press the corresponding buttons using threatening intention judgment, while eventrelated potentials (ERPs) were recorded. According to a cluster permutation test, we analyzed N190, N2, EPP (early posterior positivity), and P3. The results showed there was a positive correlation between the amplitude of the EPP induced by three kinds of body actions and the reaction time of the task. The results also revealed that when the deflection of EPP was less positive, the reaction time was shorter. We suggest that EPP might be useful as an index of body intention processing of the brain. The current study revealed that intention perception of body actions modulates brain processing.

Keywords: body action, threatening intention, early posterior positivity, reaction time, event related potentials

\section{INTRODUCTION}

The issue of threat detection was vital in many situations during human phylogenesis to allocate attention to threats to facilitate adequate reactions in due time (Feldmann-Wüstefeld et al., 2011). Threats are usually thoroughly processed (Williams et al., 2006), and they prepare the body for greater action tendency (Schutter et al., 2008; van Loon et al., 2010; Borgomaneri et al., 2014) and for quick actions (Coombes et al., 2005; Blanchard et al., 2011). This phenomenon has been shown to occur in response to threat-related words (Fox et al., 2001), pictures (Yiend and Mathews, 2001), faces, or movements (Fox et al., 2001, 2002; Borhani et al., 2015; de Valk et al., 2015). For example, anger compared with neutral body movements not only induced larger peak amplitudes on early body-related components, such as the N190 (Thierry et al., 2006; van Heijnsbergen et al., 2007; de Gelder et al., 2015), EPN (early posterior negativity) (Price et al., 2012; Borhani et al., 2015), but also boosted later potentials that reflected decision making processes (Liddell et al., 2004; Borhani et al., 2015). Furthermore, extensive studies on the processing of body movements showed that anger or fear body movements yielded automatic defense responses, such as fight, flight, and freeze (Tamietto and De Gelder, 2010; Pichon et al., 2012; de Gelder, 2013; Kveraga et al., 2015). 
Thus, humans could be well adapted to the quick recognition of another's threat and could mount an adequate response to it.

The behavioral evidence from threat detection has found that anger, rather than negative emotion, triggers faster cognitive processes (Juth et al., 2005; Schimmack and Derryberry, 2005; Frischen et al., 2008). For example, schematic angry, happy, and neutral faces were used to test the hypothesis that humans preferentially orient their attention toward a threat, which showed that angry faces were more quickly and accurately detected than other negative emotion faces (sad or "scheming") (Feldmann-Wüstefeld et al., 2011). Other non-verbal stimuli, such as body actions, convey information, and have also demonstrated this phenomenon. Gilbert et al. (2011) first adopted a visual search paradigm to explore attention bias toward body movements. The results showed that individuals detected the discrepant angry movement in a negative emotion crowd at a faster speed, whereas they detected the discrepant negative emotion movement in an angry crowd at a slower speed. The results also showed that the human brain had an attentionholding advantage of threat (Fox et al., 2001; Azarian et al., 2016). Thus, we inferred that other information, but not negative emotion signals, could explain the induced threat detection advantage.

Emotional body movements, such as anger or fear, have emotion-intention duality (i.e., emotion is intertwined with the intention in the emotional body movements) (Schupp et al., 2004; de Gelder, 2006; Azarian et al., 2016). Thus, the human brain not only processes emotional signals, but also processes the intention conveyed by such body movements (Heberlein, 2008; Steel et al., 2015; Okruszek, 2018). Considering the interference effect of prior and automatic processing of emotion relative to intention (Chouchourelou et al., 2006; Rellecke et al., 2012), it remains unclear whether an electrophysiological component is specifically responsible for processing threatening intention from body movements.

Few studies have directly explored the underlying neurophysiological characteristics of threatening intention processing, although the results of above behavioral experiments have partly conformed to this reasoning. In a neurophysiological study on intention (Schupp et al., 2004), participants were asked to observe human angry and fear faces in the absence of any task or instruction to evaluate the categorization of the expressions. The results showed that EPN bilaterally pronounced relative negativity over temporo-occipital areas, showing significant differences in different types of facial stimuli. Thus, the study suggested that the EPN represents enhanced processing of threat intention. However, in that study, there were at least two factors that need to be clarified. First, extensive studies have shown that the EPN reflects sensory encoding of emotional stimuli (Junghöfer et al., 2001; Uusberg et al., 2013; Borhani et al., 2015), and in the study of Schupp et al. (2004), the task did not require participants to identify emotion; however, EPN still emerged in the electroencephalogram (EEG). Thus, we speculated that the EPN could be an automatic process indicator of emotional stimuli, independent of task instruction. Our inference was also in line with two-stage models of visual perception that associated the EPN with initial relevance detection (Uusberg et al., 2013).
This view also suggested that the EPN is not suitable to explore the brain sensitivity to intention conveyed by emotional body movements. Second, it is easy to overlook the results of the study of Schupp et al. (2004). The authors showed that compared with friendly and neutral faces, threatening faces induced a significant positive deflection in the time windows of $220-320 \mathrm{~ms}$ on centroparietal sites. Thus, we inferred that this component could represent intention processing, and we called this component EPP (early posterior positive). Based on the interaction effect of emotion with intention processing in the emotional body movements, and the importance of detecting intention for the conduct of social life, the current study was designed to investigate, using the high temporal resolution of ERPs, whether there was a special electrophysiological component responsible for the intention decoding of threatening body movements.

In addition, the current study is also the first to explore the behavioral characteristics induced by threatening body movements. Using a new experimental paradigm, this study investigated the brain processing toward highly threatening (HT), moderately threatening (MT), and non-threatening (NT) body movements, as played by two male actors. We opted for these actions for two reasons. First, in contrast to emotional body movements, threatening body movements only transmit explicit intention without transmitting any emotional signals. Second, male actors were recruited exclusively because previous research has shown they evoke fewer affective responses than female actors (Kret et al., 2011). This study aimed to investigate whether EPN could also be induced by threatening intention and if EPP could be used an index of intentional action processing. In summary, we expected that EPP would emerge as a special component in the brain processing stream that is sensitive to threatening intention.

\section{MATERIALS AND METHODS}

\section{Participants}

Twenty-seven participants (15 male) took part in the experiment. The mean age was 22.21 [standard deviation $(S D)=2.36$ ], with ages ranging from 18 to 24 years old. The participants were recruited at the psychology laboratory of the Shanghai Normal University, Shanghai, China. They filled out an informed consent form and were debriefed after the experiment, for which they obtained course credit. Participants had no neurological or psychiatric history, and were right-handed and had normal or corrected-to normal vision. The study was performed in accordance with the Declaration of Helsinki and approved by Shanghai Normal University ethical committee.

\section{Materials Construction}

This study used experimental materials showing intentional actions instead of emotional body movements. There were three steps to ensure no explicit emotion in our intentional action pictures. At step 1, one semiprofessional male actor was hired and instructed to play various actions in three different scenarios (attacking another person, dancing with a lover, and harming others with a knife), which were recorded by a camera located at an angle of $90^{\circ}$ relative to directly facing the actor. Finally, 
30 pictures were required in which the figure faced another with the movements of stabbing (HT), fighting (MT), or dancing (NT) serving as the intentional actions. These stimuli were rated for threat on 7 -point scales ( $1=$ "high threat"; $7=$ "low threat") using an online sample $[N=91,53$ female, mean deviation of age $\left(M D_{\text {age }}\right)=25, S D=2.29$, range $=19-35$ ] on Wenjuanxing ${ }^{1}$. The results showed that stabbing actions were rated as more threatening $(M=1.60, S D=0.28)$ than fighting $(M=2.22$, $S D=0.43)[t(89)=37.344, p<0.001, d s=5.95]$ and dancing $(M=5.12, S D=0.38)$ [ $t(89)=47.586, p<0.001, d s=7.65]$; fighting actions were rated as more threatening than dancing $[t(89)=26.684, p<0.001, d s=3.75]$.

In step 2, we randomly chose 30 emotional movement pictures (fear, anger, and happy) from the BEAST stimulus database (de Gelder and Van den Stock, 2011) (Angry: F01AN, F02AN, F03AN, F04AN, F05AN, F06AN, F07AN, F08AN, F09AN, and F10AN; Fear: F01FE, F02FE, F03FE, F04FE, F05FE, F06FE, F07FE, F08FE, F09FE, and F10FE; Happy: F01HA, F02HA, F03HA, F04HA, F05HA, F06HA, F07HA, F08HA, F09HA, and F10HA). In step 3, we compared two sets of pictures to demonstrate that the pictures we made are different from the emotional pictures and have no emotional signals. All these pictures were mixed into a set and silhouetted using GIMP 2 . We then invited 92 participants to classify them according to emotion by pressing the corresponding key (1: angry, 2: disgust, 3: fear, 4: happy, 5: sad, respectively). The final results showed that the accuracy of identifying the emotion shown in the pictures obtained from the BEAST database was about 91\% (angry), 95\% (fear), and 96\% (happy), and the mean reaction time was $510 \mathrm{~ms}$ (angry), $525 \mathrm{~ms}$ (fear), and $499 \mathrm{~ms}$ (happy). However, for intention pictures, the mean reaction time was $980 \mathrm{~ms}$ and the probability of HT pictures being categorized as anger, fear, sadness, happiness, or disgust were $33,30,23,10$, and $4 \%$, respectively. The probability of the MT pictures being categorized as anger, fear, sadness, happiness, or disgust were $31,32,22,12$, and $3 \%$, respectively. The probability of the NT pictures being categorized as anger, fear, sadness, happiness, or disgust were 25, $21,10,25$, and $19 \%$. Thus, the emotional categorization of the intentional actions occurred by chance, which showed that that intentional pictures did not convey any specific emotion. All of these results testified that intentional body movements from step 1 did not convey any explicit emotion.

\section{Subjective Rating of the Action Intention}

Thereafter, the 30 intentional pictures (including $10 \mathrm{HT}$, $10 \mathrm{MT}$, and $10 \mathrm{NT}$ ) were equated for luminance and root mean square contrast (not including the gray background in calculation), and validated the threat intention of these body actions. Thirty students majoring in psychology evaluated the threatening intention (rated on 7-point scale, from 1 "the least threatening" to 7 "the most threatening") and the friendly intention (rated on 7-point scale, from 1 "the least friendly" to 7 "the most friendly") for the action pictures under all three conditions. Stimulus was presented in a

${ }^{1}$ https://www.wjx.cn/

${ }^{2}$ https://www.gimp.org/ pseudo random sequence, and Table 1 shows the results of all three conditions. One-way analysis of variance (ANOVA) showed that the main effect of different actions on threatening intention rating was significant, $F(2,88)=110.127, p<0.001$, $\eta_{\mathrm{p}}^{2}=0.227$. More specifically, stabbing was perceived as the most threatening in all kinds of actions $\left[t(29)_{\text {stabbing-fighting }}=59.351\right.$, $\left.p<0.001 ; t(29)_{\text {stabbing-dancing }}=89.732, p<0.001\right]$, fighting was perceived as more threatening than dancing actions $\left[t(29)_{\text {fighting-dancing }}=35.668, p<0.001\right]$. Meanwhile, the main effect of the different kind actions on the friendly intention rating was also significant, $F(2,88)=64.359, p<0.001, \eta_{p}^{2}=0.239$. Dancing actions, in which there was no significant difference between MT and HT, were perceived as friendlier than the other two kinds of actions $\left[t(29)_{\text {dancing-fighting }}=21.259, p<0.001\right.$; $\left.t(29)_{\text {dancing-stabbing }}=52.722, p<0.001\right]$. Fighting was perceived as friendlier than stabbing $\left[t(29)_{\text {fighting-stabbing }}=17.645\right.$, $p<0.001$ ], which was perceived least the friendly of all the kinds of actions.

\section{Procedure}

A new intention categorization task was conducted. Participants were seated in a darkened and sound-attenuated room, and received the presentation of stimuli on a $19^{\prime \prime}$ monitor (1024 pixels $\times 1024$ pixels) with a gray background. All picture stimuli had the same size $(10 \mathrm{~cm} \times 10 \mathrm{~cm})$. The viewing angle was set at about $9.5^{\circ} \times 9.5^{\circ}$, and the picture size was 512 pixels $\times 512$ pixels, and the image sampling rate was 72 ppi. After participants read the information brochure and signed the informed consent, and then given verbal instructions. The distance between the participants and screen was $80 \mathrm{~cm}$, a distance at which all participants could comfortably press the keyboard. HT, MT, and NT body actions were randomly presented on the computer screen using E-prime 2.0 (Psychology Software Tools, Inc., 2012). The participants pressed the "J" button with the index finger for threatening movements and the "L" button with the ring finger for NT movements.

The trial started with the presentation of a fixation ("+") in the center of the screen for 500-800 ms randomly. Second, a blacksilhouetted body action appeared on the screen for $800 \mathrm{~ms}$. In this phase, participants were instructed to press buttons. After that, a feedback screen displaying the reaction time and accuracy was sustained for $1500 \mathrm{~ms}$, and the inter-trial interval was 1000$1500 \mathrm{~ms}$ randomly (see Figure 1). Participants were asked to focus on the central fixation point and to avoid head movement

TABLE 1 | Mean threat and friendliness ratings when view non-threatening, moderately, and highly threatening body actions.

\begin{tabular}{|c|c|c|c|c|c|c|}
\hline \multirow{2}{*}{$\begin{array}{l}\text { Dependent } \\
\text { Measure }\end{array}$} & \multicolumn{2}{|c|}{$\begin{array}{l}\text { Dancing images } \\
\text { (NT) }\end{array}$} & \multicolumn{2}{|c|}{$\begin{array}{l}\text { Fighting images } \\
\text { (MT) }\end{array}$} & \multicolumn{2}{|c|}{$\begin{array}{c}\text { Stabbing } \\
\text { images (HT) }\end{array}$} \\
\hline & $M$ & $S D$ & $M$ & $S D$ & $M$ & $S D$ \\
\hline $\begin{array}{l}\text { Threatening } \\
\text { rating }(1-7)\end{array}$ & 1.72 & 1.01 & 5.01 & 2.45 & 6.84 & 1.16 \\
\hline Friendliness & 4.25 & 1.14 & 1.23 & 1.73 & 1.08 & 0.62 \\
\hline
\end{tabular}




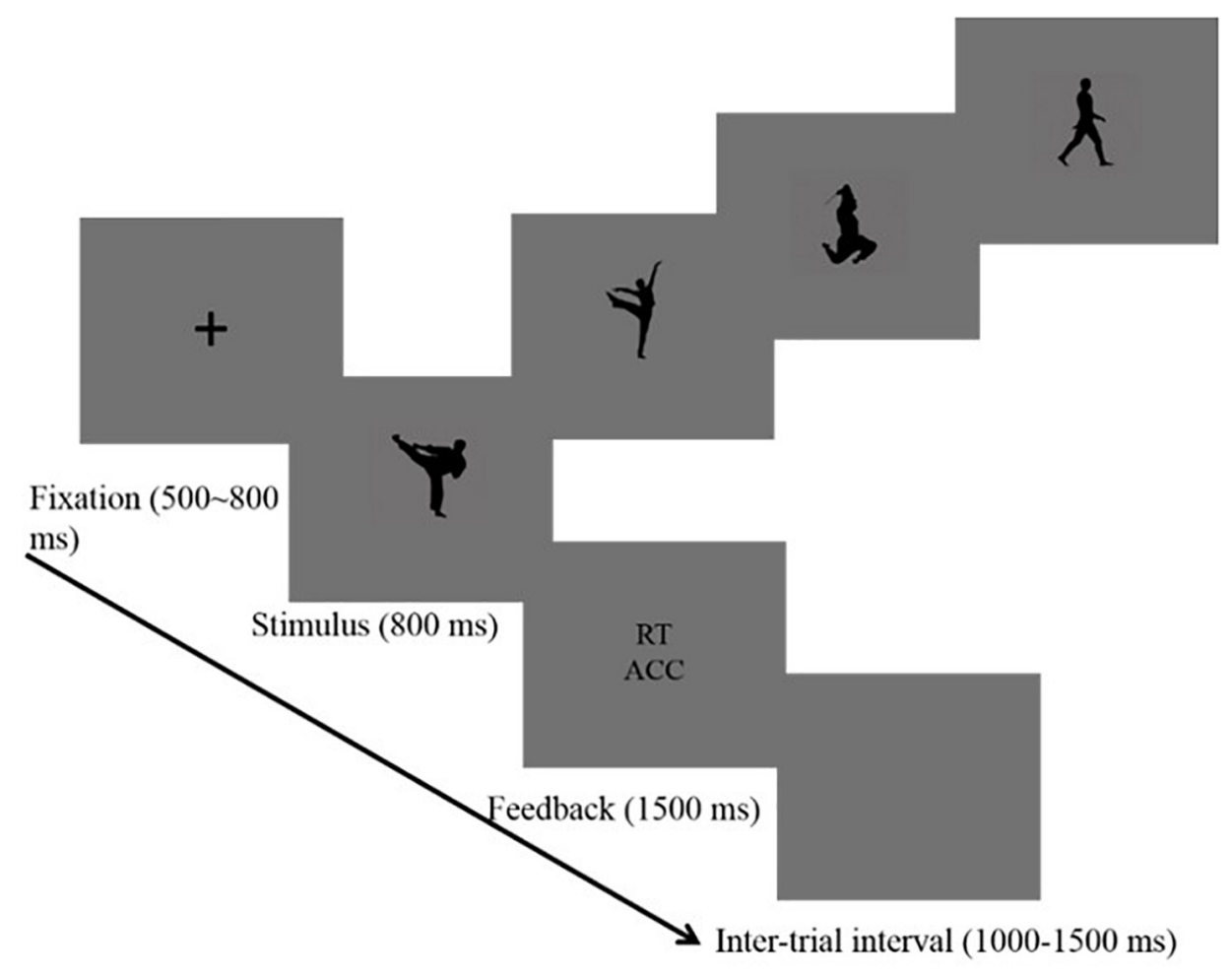

FIGURE 1 | Procedure used in the categorization task that shows the sequence of events within a trial.

or eye blink as much as possible. Reaction times were measured and the EEG was recorded. There were eight blocks with a total of 480 trials using a random order. In each block, 60 trials were randomly presented ( 20 trials $\times 3$ body stimuli: $\mathrm{HT}, \mathrm{MT}$, and NT). A self-paced break was provided at each block. The trial number in this study met the requirement that the number of trials in ERP studies should be high enough to reach a high signal-to-noise (SNR) (Luck, 2014; Thigpen et al., 2017).

\section{Event-Related Potential Recording and Analysis}

Electroencephalography (EEG) was recorded from 64 scalp sites using Ag/AgCl electrodes mounted on an elastic cap, according to the extended international 10-20 system (Neuroscan, Sterling, VA, United States), with the references on the left and right mastoids, and a ground electrode on the medial frontal aspect. Vertical electrooculograms (EOGs) were recorded supraorbitally and infraorbitally at the left eye. The horizontal EOG was recorded as the left versus the right orbital rim. EEG and EOG activity was amplified using a DC $100 \mathrm{~Hz}$ bandpass and continuously sampled in the $500 \mathrm{~Hz} / \mathrm{channel}$. The data was low pass filtered offline at $30 \mathrm{~Hz}(24 \mathrm{~dB} /$ oct $)$, and all electrode impedances were maintained below $5 \mathrm{k} \Omega$. Data pre-processing was performed in Neuroscan (Scan 4.4). An automated eyemovement correction program was used before artifact rejection (Gratton et al., 1983), based on the Least Mean Squares algorithm. ERP averages were computed off-line. Trials with remaining EOG artifacts (mean EOG voltage exceeding $\pm 80 \mathrm{mV}$ ), amplifier clipping artifacts, or peak-to-peak deflection exceeding $\pm 80 \mathrm{mV}$, were excluded from averaging. The remaining epochs (mean: 163 epochs per body stimulus condition) were averaged separately for each participant and each body stimulus condition. ERP waveforms were time locked, and the average epoch was $1000 \mathrm{~ms}$, including a $200 \mathrm{~ms}$ pre-stimulus baseline. The current study was the first to explore the electrophysiological characteristics of threat intention conveyed by body action, and thus we analyzed the brain information processing stream in the ERP results part.

Considering that this study was exploratory, a cluster-based permutation test was conducted using the FieldTrip toolbox (Oostenveld et al., 2011) to perform a planned comparison between pairs of conditions. The cluster-based permutation test uses non-parametric statistics to capture ERP effects without prior assumptions about their scalp distribution and latency range (Maris and Oostenveld, 2007). Each compete set of data was randomly divided into two subsets and a new summed $t$-value was calculated, which was repeated 10,000 times. The initial alpha value for cluster formation was set at alpha $<0.01$ to reduce the likelihood of large clusters spanning the entire dataset (Mensen and Khatami, 2013).

As shown by the ERP's grand averaged waveforms, according to the cluster permutation test (Figures 2-4), the ERPs elicited by the three conditions showed prominent differences from each other and these differences were the largest at the central and frontal sites (see Figures 5, 6). N2 over a frontal-central region across the scalp is attributed to attention resource for different negative pictures and threat detection (Thorpe et al., 1996). 


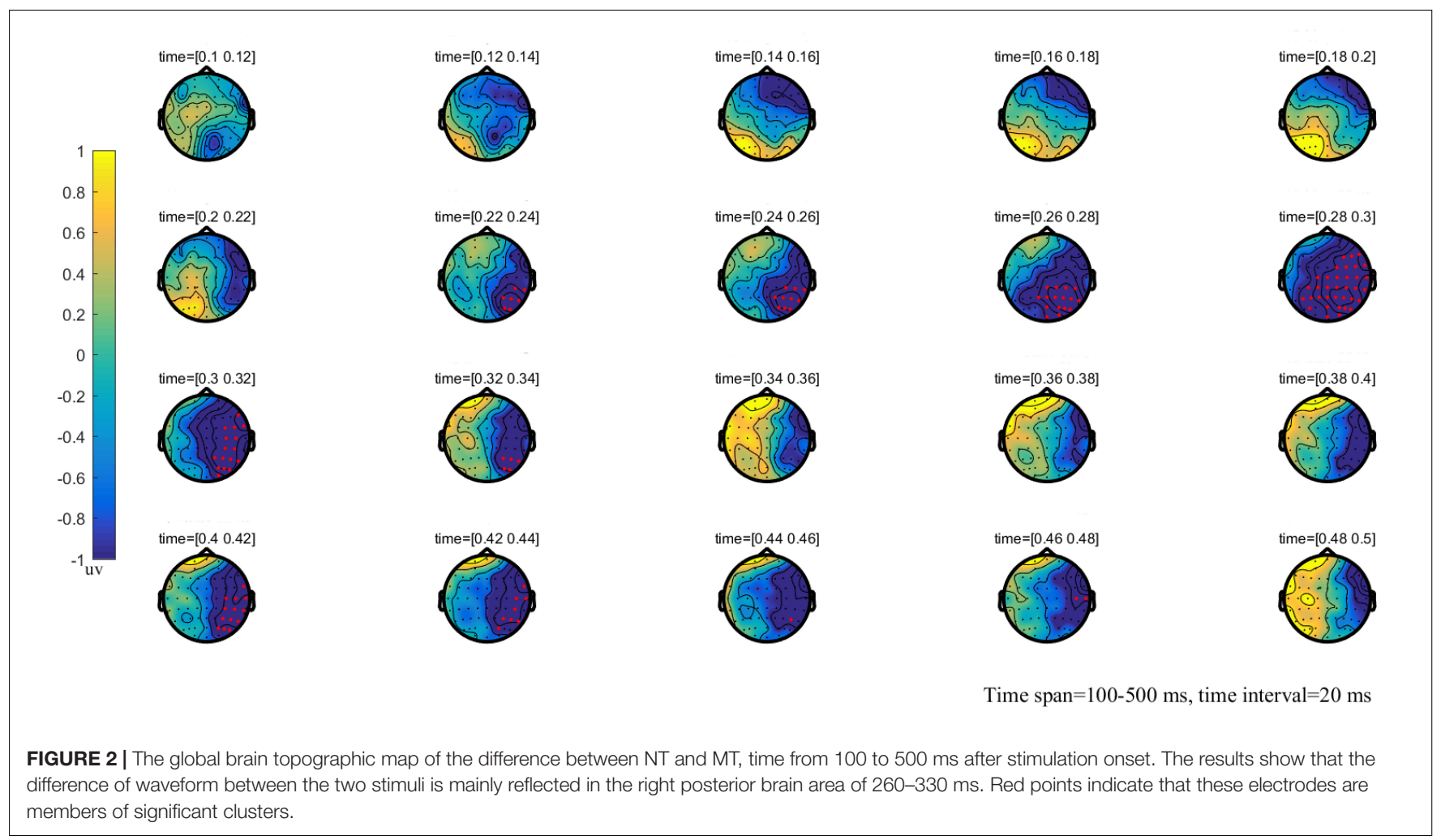

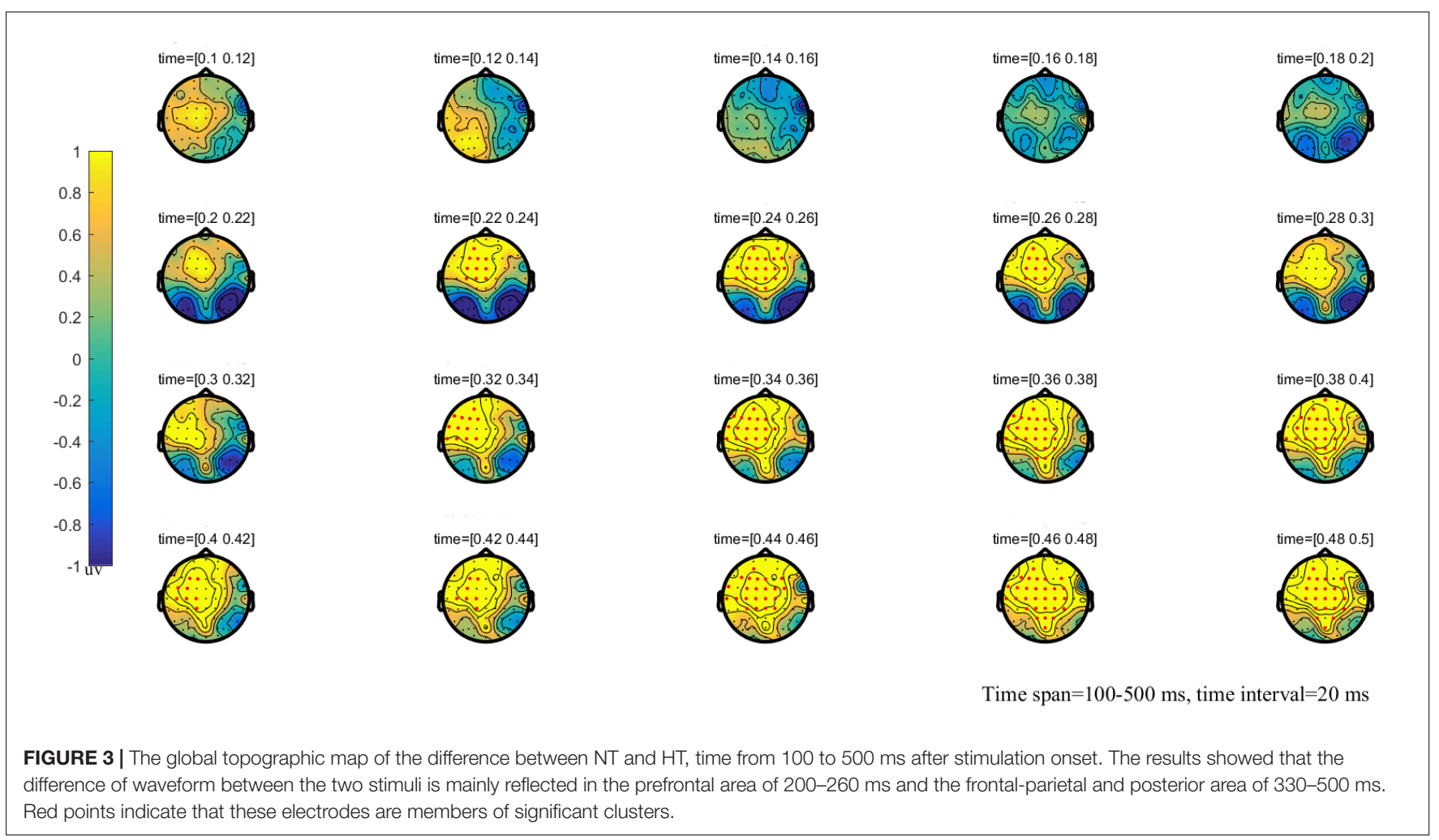

In addition, previous studies have shown that the anterior N2 component also represents the allocation of attention to novel or threatening stimuli (Yuan et al., 2007). During the later stages of stimulus presentation, $\mathrm{P} 3$, a positive-going centro-parietal ERP beginning approximately $300 \mathrm{~ms}$ after stimulus onset, was detected, reflecting cognitive evaluation of a stimulus' meaning 

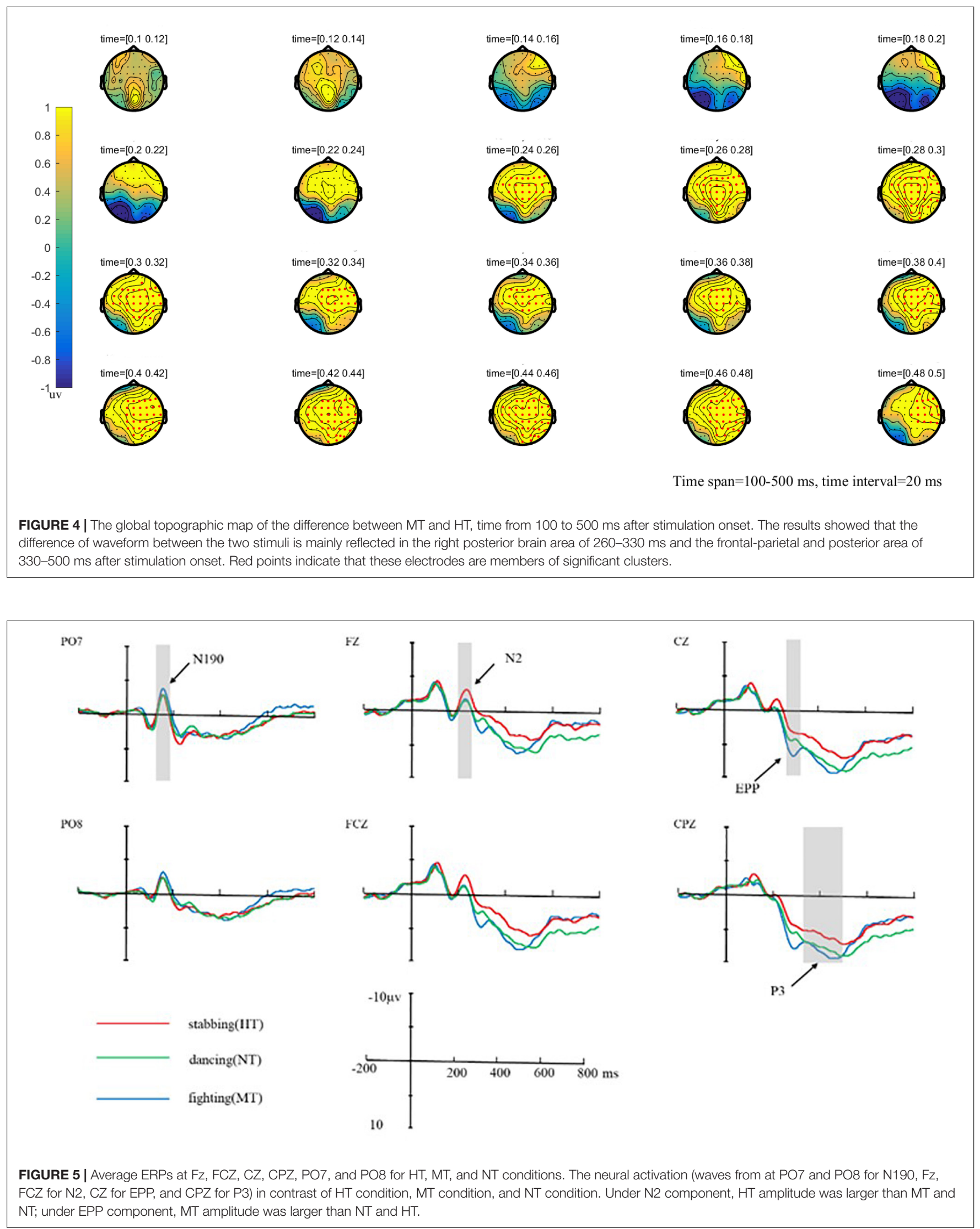

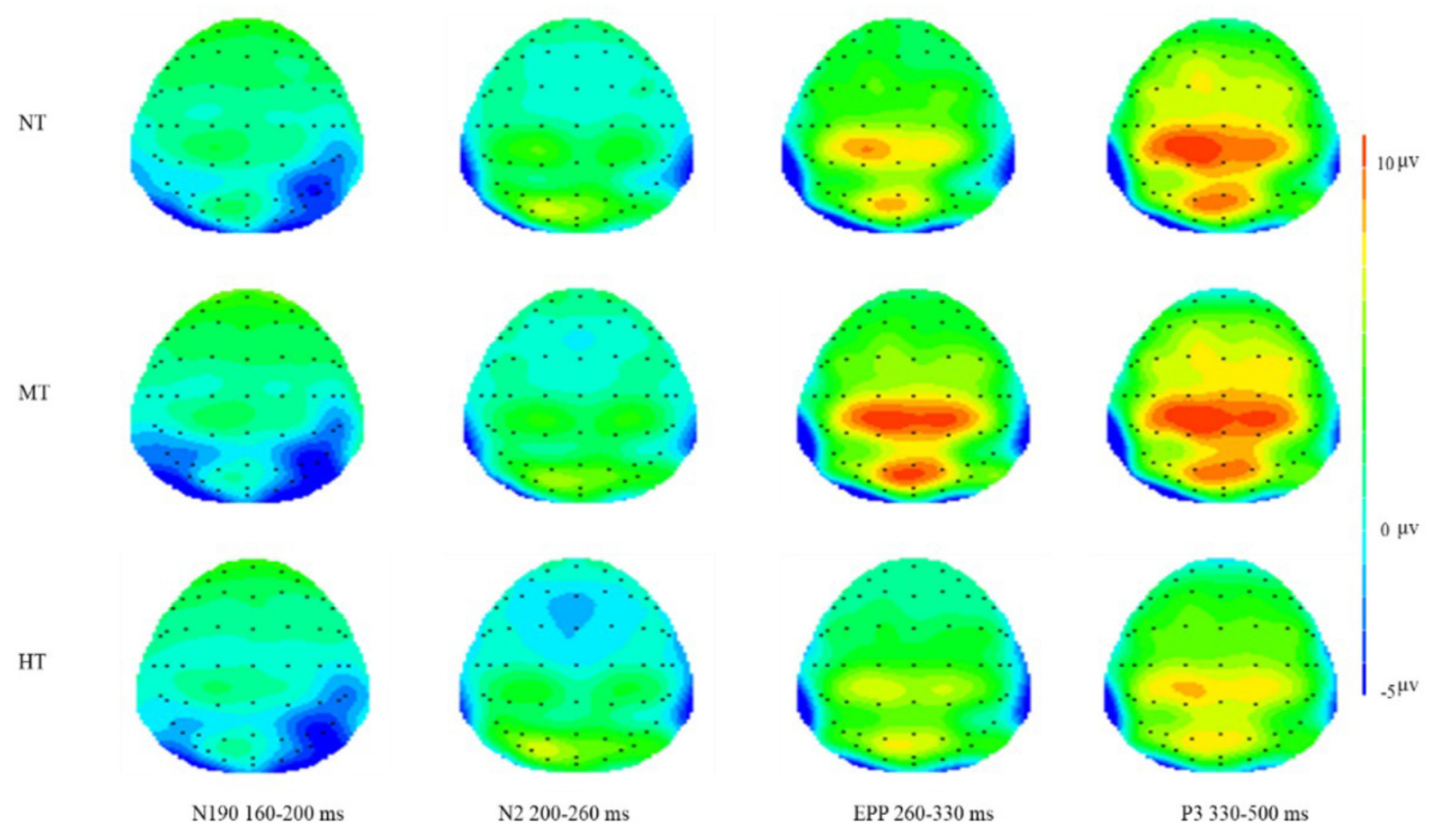

FIGURE 6 | Topographical maps of three conditions on N190, N2, EPP, and P3. Significantly larger frontal N2 amplitude occurred in the HT condition and significant central EPP amplitude occurred in the MT condition.

(Polich, 2007; Wei et al., 2016) and the elaborated processing of stimulus meaning (Hajcak and Olvet, 2008; Weinberg and Hajcak, 2010). Thus, we selected the following 12 electrode sites for statistical analysis: FZ, F3, F4, FC3, FC4, FCZ, C3, C4, CZ, CP3, CP4, CPZ (12 central-parietal and frontal sites) for EPP, and N2 and P3 according to the time and topography of these components; in addition, we selected PO7 and PO8 (two parietal-occipital sites) for N190 and EPN. Thierry et al. (2006) found a negative component peaking at $190 \mathrm{~ms}$ post-stimulus onset (N190), reflecting the structural visual encoding of bodies. At a later stage of visual processing, salient emotional bodies modulate the amplitude of the EPN, which reflects stimulusdriven attentional capture, in which relevant stimuli are selected for further processing (Schupp et al., 2004; Calvo and Beltrán, 2014). Thus, the N190 amplitude was quantified as the mean amplitude in a window of 160-200 ms of each target site. The mean amplitudes of N2 (200-260 ms), EPN (200-400 ms), EPP (260-330 ms), and P3 (330-500 ms) components were also analyzed using the above method.

The main aim of this study was to explore whether EPP is an electrophysiological indicator of intention processing, and whether this component would trigger an adaptive action response. A two-way repeated measure analysis of variance (ANOVA) was conducted for the amplitude of each component. ANOVA factors were threat intention (three levels: HT, MT, and NT) and electrode site (12 sites for N2, EPN, EPP and P3; two sites for N190). The dependent variable was the average amplitude of the time period. The significance level for all ANOVAs was set at 0.05. In all cases, these components were statistically evaluated using SPSS (version 20.0) and Greenhouse-Geisser correction was used when the hypothesis of sphericity was not met. For significant main effects or interactions, Bonferroni corrected $p$-values were reported for post hoc comparisons.

\section{RESULTS}

\section{Behavioral Results}

The mean accuracy of three conditions was 96\% (HT), 93\% (MT), and $93 \%(\mathrm{NT})$, respectively. This showed that participants could perceive and classify the action intentions effectively. The results showed a main effect of threatening intention, $F(2,52)=17.123$, $p=0.002, \eta_{\mathrm{p}}^{2}=0.233$, with significant faster reaction times following $\mathrm{HT}(M=461 \mathrm{~ms}, S D=33 \mathrm{~ms})$ as compared with MT $(M=496 \mathrm{~ms}, S D=26 \mathrm{~ms}, p<0.001)$ and NT $(M=477 \mathrm{~ms}$, $S D=37 \mathrm{~ms}, p=0.003)$. A Bonferroni-corrected pairwise comparison showed that the difference between MT and NT was significant (Mean Difference $=19 \mathrm{~ms}, S E=4.23, p=0.019$ ). The behavioral results showed that HT induced the fastest response, followed by NT, and the response induced by MT was slowest.

\section{Cluster-Based Permutation Test}

The results of the cluster permutation test are shown in Figures 2-4, which revealed several significant clusters in different conditions. Note that significant clusters represent sampling points with spatial and temporal adjacency. The contrast between NT and MT elicited a significant negative 
cluster, with a time window during 260-330 ms typical of an EPP (sum- $T=-424.86, p<0.002$ ). In the comparison between NT and HT, HT also showed an early cluster (from 200 to $260 \mathrm{~ms}$; sum- $T=164.8, p=0.002$ ), which could be considered as an N2, and a significant positive cluster $(330-500 \mathrm{~ms}$; sum- $T=775.5$, $p \leq 0.001$ ), which could be considered as a P3. The significant cluster was a positive one exhibited in the MT/HT contrast during 260-330 ms (sum- $T=875.18, p=0.018$ ), which was identified as an EPP, and during 330-500 $\mathrm{ms}$ (sum- $T=775.5, p=0.002$ ), which was identified as a P3. The scalp distributions of these clusters informed our selection of several ERP components that fell within the clusters (see Figures 5, 6), which we investigated using ANOVAs. We also report ANOVAs on some components of a priori interest.

To summarize, statistically reliable N2, EPP, and P3 clusters were observed only in the comparisons among NT/MT/HT. HT exhibited a higher N2 and MT exhibited a higher EPP.

\section{ERP Analysis}

We analyzed the brain information processing stream (including N190, N2, EPN, EPP, and P3) in the ERP results. As shown in Figures 5, 6, N190, N2, EPP, and P3 components were elicited by all three conditions. In this study, a significant main effect of threat intention was found for N190 $[F(2,52)=10.623$, $\left.p=0.003, \eta_{\mathrm{p}}^{2}=0.422\right], \mathrm{N} 2[F(2,52)=15.623, p<0.001$, $\left.\eta_{\mathrm{p}}^{2}=0.435\right], \operatorname{EPP}\left[F(2,52)=55.623, p<0.001, \eta_{\mathrm{p}}^{2}=0.435\right]$, and P3 $\left[F(2,52)=28.648, p=0.006, \eta_{\mathrm{p}}^{2}=0.222\right]$ amplitudes.

\section{N190 Response}

For the N190 response, mean amplitudes during the interval from 160 to $200 \mathrm{~ms}$, a two-way repeated measures ANOVA was conducted for the amplitude of this component. ANOVA factors were threat intention (HT, MT, and NT) and electrode site (two sites, PO7, PO8). The results revealed that the main effect of intention processing was significant $\left(M_{\mathrm{HT}}=-2.520, S D=0.861\right.$, $\left.M_{\mathrm{NT}}=-2.514, S D=0.933 ; M_{\mathrm{MT}}=-3.278, S D=0.870\right)$, $F(2,52)=10.623, p=0.003, \eta_{\mathrm{p}}^{2}=0.422$. Post hoc analyses showed a significantly larger N190 amplitude in response to MT compared with HT $(p<0.01)$ and NT $(p<0.01)$. The interactions between threatening conditions and electrodes were not significant, $F(2,52)=1.317, p=0.16$. In addition, there was also no significant difference found between the NT and HT.

\section{N2 Response}

The potential component at 200-260 ms was analyzed through within-participant two-way ANOVA with intention (HT, MT, and NT) and electrode site (12 sites) This result revealed that main effect of threat intention was significant $\left(M_{\mathrm{HT}}=-1.17\right.$, $S D=0.563 ; M_{\mathrm{NT}}=-0.635, S D=0.647 ; M_{\mathrm{MT}}=-0.622$, $S D=0.570), F(2,52)=15.623, p<0.001, \eta_{\mathrm{p}}^{2}=0.435$. Post hoc analyses showed a significantly larger $\mathrm{N} 2$ amplitude in response to HT compared with all other stimuli (all $p<0.01$ ). There was no significant difference found between the NT and MT $(p=0.099)$. Additionally, a main effect of amplitude at electrode sites was significant for $\mathrm{N} 2\left[F(11,286)=255.75, p<0.01, \eta_{\mathrm{p}}^{2}=0.135\right]$. The largest N2 amplitudes were recorded at the frontal electrode sites [e.g., Fz, F3, F4] and all anterior sites displayed a larger
$\mathrm{N} 2$ than the posterior sites. The interactions between threatening conditions and electrodes was not significant, $F(22,572)=1.370$, $p=0.56$.

\section{EPN Response}

The potential component of EPN at 200-400 ms was analyzed using two-way ANOVA with threat intention (HT, MT, and NT) in PO7 and PO8 electrode sites. This result revealed no significant difference among three conditions, $F(2,52)=3.389, p=0.078$, $\eta_{\mathrm{p}}^{2}=0.122$. No other comparisons were significant (all $p>0.69$ ).

\section{EPP Response (260-330 ms)}

Event-related potential responses are shown in Figures 5, 6. The results of two-way ANOVA showed that the intention main effect was significant $\left(M_{\mathrm{HT}}=0.848, S D=0.453 ; M_{\mathrm{NT}}=1.998\right.$, $\left.S D=0.443 ; M_{\mathrm{MT}}=3.811, S D=0.580\right), F(2,52)=15.623$, $p<0.001, \eta_{\mathrm{p}}^{2}=0.345$. Post hoc analyses showed a significantly larger EPP amplitude in response to MT compared with the other kinds of stimuli (all $p<0.01$ ). In addition, the amplitude of NT reached a more significantly positive deflection than HT $(p=0.002)$. Additionally, a main effect of amplitude at electrode sites was significant for $\operatorname{EPP}[F(11,286)=342.29, p<0.01$, $\left.\eta_{\mathrm{p}}^{2}=0.135\right]$. The interaction between threatening conditions and electrodes was significant, $F(22,572)=9.123, p<0.001$.

\section{P3 Response}

For the P3 response amplitudes during the interval from 330 to $500 \mathrm{~ms}$, the within-participant two-way ANOVA with threat intention (HT, MT, and NT) and electrode sites (12 sites) revealed that the intention main effect was significant $\left(M_{\mathrm{HT}}=2.742\right.$, $\left.S D=0.589, M_{\mathrm{NT}}=5.335, S D=0.763 ; M_{\mathrm{MT}}=5.278, S D=0.870\right)$, $F(2,52)=18.648, p=0.0026, \eta_{\mathrm{p}}^{2}=0.222$. Post hoc analyses showed a significantly smaller P3 amplitude in response to HT compared with MT and NT (all $p<0.01$ ). There was no significant difference found between the NT and MT $(p=0.164)$. Additionally, a main effect of amplitude at electrode sites was significant for the P3 component $[F(11,286)=179.34$, $\left.p<0.01, \eta_{\mathrm{p}}^{2}=0.126\right]$. The interaction between threatening conditions and electrodes was significant, $F(22,572)=5.154$, $p<0.001$.

\section{Correlations}

We calculated the correlations between reaction time and the amplitude of EPP, N190, N2, and P3 from the 27 participants. We found significant correlation between EPP amplitude and reaction time under HT $(r=0.787)$, NT $(r=0.671)$, and MT $(r=0.720)$ conditions (all ps $<0.001)$. Individuals with a lower EPP amplitude showed a faster response toward the threatening intention (Figure 7). The linear relationships among the RT (reaction time) with N190, N2, and P3 were also calculated; however, no significant correlation was found between them and the RT measure (all $p>0.10$ ).

\section{DISCUSSION}

In previous studies on intention processing, researchers mainly used emotional body movements as the experiment object. 
HT

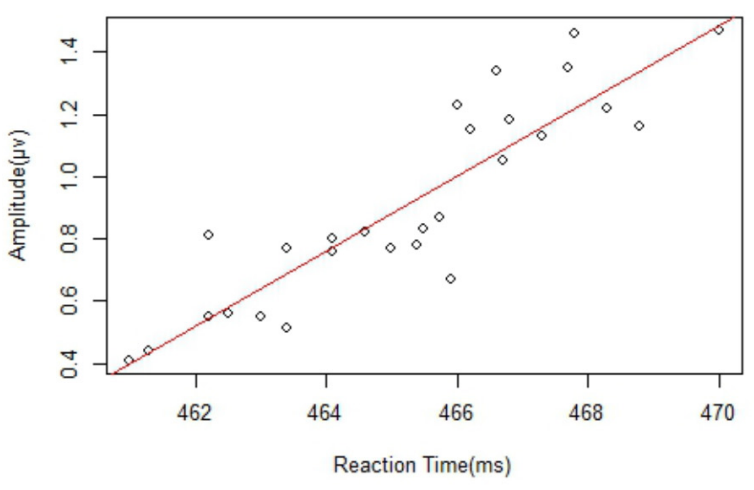

NT

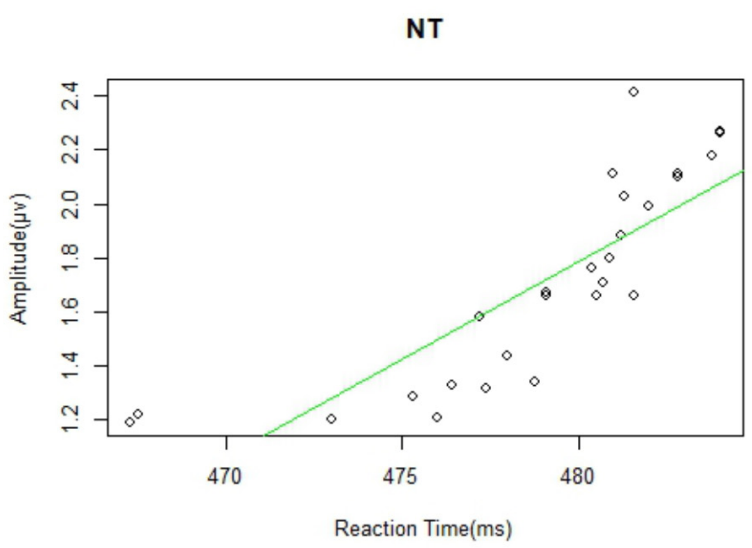

MT

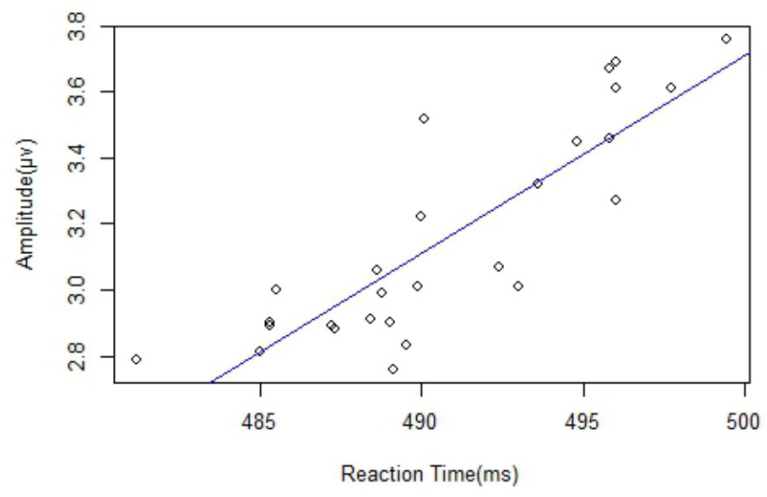

FIGURE 7| There were significant correlations between EPP and reactive time under different action conditions.

However, because of the emotion and intention duality of emotional body movements, the perception and response of the human brain to intentions is always disturbed by emotions. Thus, we could not paint a conclusive picture about the relationship between intention processing and brain components. This study used threatening body actions without explicit emotions, but with obvious intentions, as experimental subjects. The behavioral results showed that participants responded faster to HT than to MT and NT stimuli, meanwhile, they responded faster to NT pictures than to MT pictures. More importantly, the amplitude of EPP in each condition was positively correlated with RT. Thus, EPP could be used an index of body intention processing of the brain.

At approximately $200-260 \mathrm{~ms}$ after stimulus onset, obvious $\mathrm{N} 2$ activity was generated at the frontal brain region in all three conditions, and larger N2 amplitudes were observed for the HT condition than for the MT and NT conditions over a frontal-central region across the scalp. N2 activation is indicative of attention resource for different negative pictures and threat detection (Thorpe et al., 1996). In addition, previous studies have shown that the anterior $\mathrm{N} 2$ component also represents the allocation of attention to novel or threatening stimuli (Yuan et al., 2007). Thus, our finding of larger N2 amplitudes in the HT condition is likely an index of a rapid attention allocation process that attends to threatening content (Li et al., 2005). Compared with the MT and NT pictures, the HT pictures often included salient threatening content (e.g., knife and gun), which has been shown to recruit human attention resources rapidly and automatically (Hansen and Hansen, 1988; Li et al., 2005). There was no significant difference between MT and NT. It is likely that these stimuli were just body actions. Accordingly, in the present study the detection of salient threatening information was facilitated.

Previous studies have shown that the perception of social intention is directly related to body actions; even for a simple gesture and its action, kinematics has shown differences between the conditions with and without communicative intentions (Pierno et al., 2008; Sartori et al., 2009). The purpose of our research was to explore the neural characteristics of perceiving intentions and the relationship between subsequent actions. The current results showed that there was a positive correlation between the amplitude of the EPP and the RT. The lower amplitude of EPP, shorter the RT. A possible explanation for EPP is that intention (i.e., carried by fighting or stabbing) can induce attention automatic allocation, which serves to understand action intention in the current task. In contrast to N2, the EPP component was significantly larger in the MT condition than in the HT and NT condition, which indicates that the action 
intention of the MT stimuli was detected and obtained more attention resources than other stimuli. This is most likely because, at the conscious level, the brain had begun to integrate the features for intention understanding. The intention of HT could be understood easily in the N2 stage because of salient stimuli, such as a gun or knife, and thus the EPP of HT was smaller than that of MT. The intention understanding of MT could be difficult because the solitary fighting action was ambiguous and not selfrelevant. Previous studies have shown that threat bias was likely related to the direction of the threat in relation to the observer (Grèzes et al., 2013; de Valk et al., 2015). MT recruited more human attention resources for understanding the body intention, and thus, MT induced more positive drift compared with that of NT.

P3 signals the cognitive evaluation of a stimulus' meaning (Polich, 2007; Wei et al., 2016). In the present study, HT stimuli evoked the smallest P3 amplitudes. The size of the P3 amplitudes in the present experiment may reflect the degree of an individuals' decision-making. For a participant to make a correct behavioral response to the stimulus, all task-relevant information had to be evaluated correctly. It is possible that when the participants saw the gun or knife (in the N2 stage), they could identify easily that the picture should be attributed to high threat and there was no need to decode the intention of the body actions (in the EPP stage); therefore, the participants consumed fewer cognitive resources to make the decision. Therefore, it is easy to make the fastest decision under threat of the HT condition (with the least information burden), which would account for the small P3 amplitudes.

Many studies have reported a threat bias in which negative emotional body action or faces are prioritized over neutral (Hansen and Hansen, 1988; van Heijnsbergen et al., 2007; Feldmann-Wüstefeld et al., 2011; Borhani et al., 2015; de Valk et al., 2015). Our study verified those findings by showing whether perceiving explicit intentional actions could trigger faster actions or not. Our findings support the view of emotion and intention duality, and suggested that threat intention could foster action. In addition, MT stimuli triggered slower action than did NT, possibly because MT induces a complicated attention processing to intention, i.e., solitary and self-irrelevance rather than interaction and self-relevance. In other words, a stabbing picture could be perceived as a clear threat intention to the participants because a weapon or knife could assist them to understand the scene in the N2 stage; however, a solitary fight action might be more ambiguous as a source of threat intention when participants decode their intention in a limited time. Thus, we suggest that an explicit threat intention (e.g., HT) asks for immediate action and an ambiguous

\section{REFERENCES}

Azarian, B., Esser, E. G., and Peterson, M. S. (2016). Evidence from the eyes: Threatening postures hold attention. Psychon. Bull. Rev. 23, 764-770. doi: 10. 3758/s13423-015-0942-0

Blanchard, D. C., Griebel, G., Pobbe, R., and Blanchard, R. J. (2011). Risk assessment as an evolved threat detection and analysis process. Neurosci. Biobehav. Rev. 35, 991-998. doi: 10.1016/j.neubiorev.2010.10.016 threat intention (e.g., MT) requires further exploration and hence needs more processing time and a somewhat slower response. Previous studies also support this view. For instance, Hortensius et al. (2016) aimed to explore the threat detection advantage of body stimuli. In their study, fearful/angry body stimuli were directed toward or away from the observer. Singlepulse transcranial magnetic stimulation to the left primary motor cortex was applied to measure motor evoked potentials from the right abductor pollicis brevis in response to angry and fearful bodily expressions, with blurred faces. The results showed that it was easier to recognize anger directed toward the observer than that directed away from the observer. The results provided direct evidence that clear threat intention could foster fast adaptive action. In the current study, fight actions (MT) promoted slower action because the human brain has to consume more attention resource to decode the ambiguous threatening intention. In addition, enhanced responses to dance action (NT) suggested that we can readily recognize the threatunrelated but clear intention conveyed by body actions and make the correct decision. Thus, it is possible that different results may be obtained if MT stimuli become dual interaction or self-relevant, rather than solitary irrelevant threatening actions. Future studies could make use of our procedure with the aim of improving the ambiguous intention of solitary fighting actions. For example, one approach would be to present selfrelevant or interpersonal, dynamic action pictures, not a static one.

In summary, the novel experimental design made it possible to directly test brain sensitivity to intention perception. The correlation between the amplitude characteristic of EPP and reaction time indicated that the EPP might be an index of intention processing of body actions. Furthermore, the clarity of intentional actions modulates the reaction time.

\section{AUTHOR CONTRIBUTIONS}

GW collected, analyzed, interpreted the data, and wrote the paper. JL and WN helped to analyze, interpreted the data, and helped to write the paper. GW and PW conceived the project. All authors read and approved the final version of the manuscript for submission.

\section{FUNDING}

This research was supported by a grant from the National Social Science Foundation Major Program of China (17ZDA327).

Borgomaneri, S., Gazzola, V., and Avenanti, A. (2014). Temporal dynamics of motor cortex excitability during perception of natural emotional scenes. Soc. Cogn. Affect. Neurosci. 9, 1451-1457. doi: 10.1093/scan/ nst139

Borhani, K., Làdavas, E., Maier, M. E., Avenanti, A., and Bertini, C. (2015). Emotional and movement-related body postures modulate visual processing. Soc. Cogn. Affect. Neurosci. 10, 1092-1101. doi: 10.1093/scan/ nsu 167 
Calvo, M. G., and Beltrán, D. (2014). Brain lateralization of holistic versus analytic processing of emotional facial expressions. Neuroimage 92, 237-247. doi: 10. 1016/j.neuroimage.2014.01.048

Chouchourelou, A., Matsuka, T., Harber, K., and Shiffrar, M. (2006). The visual analysis of emotional actions. Soc. Neurosci. 1, 63-74 doi: 10.1080/ 17470910600630599

Coombes, S. A., Janelle, C. M., and Duley, A. R. (2005). Emotion and motor control: movement attributes following affective picture processing. J. Mot. Behav. 37, 425-436. doi: 10.3200/JMBR.37.6.425-436

de Gelder, B. (2006). Towards the neurobiology of emotional body language. Nat. Rev. Neurosci. 7, 242-249. doi: 10.1038/nrn1872

de Gelder, B. (2013). "From body perception to action preparation: a distributed neural system for viewing bodily expressions of emotion," in Social, Perceptual, and Neurophysiological Studies of Body Perception, eds K. Johnson, M. Shiffrar (Oxford: OUP)

de Gelder, B., de Borst, A. W., and Watson, R. (2015). The perception of emotion in body expressions. Wiley Interdiscip. Rev. Cogn. Sci. 6, 149-158. doi: 10.1002/ wcs. 1335

de Gelder, B., and Van den Stock, J. (2011). The bodily expressive action stimulus test (BEAST). construction and validation of a stimulus basis for measuring perception of whole body expression of emotions. Front. Psychol. 2:181. doi: 10.3389/fpsyg.2011.00181

de Valk, J. M., Wijnen, J. G., and Kret, M. E. (2015). Anger fosters action. fast responses in a motor task involving approach movements toward angry faces and bodies. Front. Psychol. 6:1240. doi: 10.3389/fpsyg.2015.01240

Feldmann-Wüstefeld, T., Schmidt-Daffy, M., and Schubö, A. (2011). Neural evidence for the threat detection advantage: differential attention allocation to angry and happy faces. Psychophysiology 48, 697-707. doi: 10.1111/j.1469-8986. 2010.01130.x

Fox, E., Russo, R., Bowles, R., and Dutton, K. (2001). Do threatening stimuli draw or hold visual attention in subclinical anxiety? J. Exp. Psychol. Gen. 130, 681-700. doi: 10.1037/0096-3445.130.4.681

Fox, E., Russo, R., and Dutton, K. (2002). Attentional bias for threat: evidence for delayed disengagement from emotional faces. Cogn. Emot. 16, 355-379. doi: 10.1080/02699930143000527

Frischen, A., Eastwood, J. D., and Smilek, D. (2008). Visual search for faces with emotional expressions. Psychol. Bull. 134, 662-676. doi: 10.1037/0033-2909.134. 5.662

Gilbert, T., Martin, R., and Coulson, M. (2011). Attentional biases using the body in the crowd task: are angry body postures detected more rapidly? Cogn. Emot. 25, 700-708. doi: 10.1080/02699931.2010.495881

Gratton, G., Coles, M. G. H., and Donchin, E. (1983). A new method for off-line removal of ocular artifact. Electroencephalogr. Clin. Neurophysiol. 55, 468-484. doi: 10.1016/0013-4694(83)90135-9

Grèzes, J., Adenis, M. S., Pouga, L., and Armony, J. L. (2013). Self-relevance modulates brain responses to angry body expressions. Cortex 49, 2210-2220. doi: 10.1016/j.cortex.2012.08.025

Hajcak, G., and Olvet, D. M. (2008). The persistence of attention to emotion: brain potentials during and after picture presentation. Emotion 8, 250-255. doi: 10.1037/1528-3542.8.2.250

Hansen, C. H., and Hansen, R. D. (1988). Finding the face in the crowd: an anger superiority effect. J. Pers. Soc. Psychol. 54, 917-924. doi: 10.1037/0022-3514.54. 6.917

Heberlein, A. S. (2008). 14. "Animacy and intention in the brain: neuroscience of social event perception," in Understanding Events: From Perception to Action, eds T. Shipley, J. Zacks (New York, NY: Oxford University Press).

Hortensius, R., de Gelder, B., and Schutter, D. J. L. G. (2016). When anger dominates the mind: increased motor corticospinal excitability in the face of threat. Psychophysiology 53, 1307-1316. doi: 10.1111/psyp.12685

Junghöfer, M., Bradley, M. M., Elbert, T. R., and Lang, P. J. (2001). Fleeting images: a new look at early emotion discrimination. Psychophysiology 38, 175-178. doi: 10.1111/1469-8986.3820175

Juth, P., Lundqvist, D., Karlsson, A., and Öhman, A. (2005). Looking for foes and friends: perceptual and emotional factors when finding a face in the crowd. Emotion 5, 379-395. doi: 10.1037/1528-3542.5.4.379

Kret, M. E., Pichon, S., Grèzes, J., and de Gelder, B. (2011). Men fear other men most: gender specific brain activations in perceiving threat from dynamic faces and bodies - an fMRI study. Front. Psychol. 2:3. doi: 10.3389/fpsyg.2011.00003
Kveraga, K., Boshyan, J., Adams, J. R. B., Mote, J., Betz, N., Ward, N., et al. (2015). If it bleeds, it leads: separating threat from mere negativity. Soc. Cogn. Affect. Neurosci. 10, 28-35. doi: 10.1093/scan/nsu007

Li, X., Li, X., and Luo, Y. J. (2005). Anxiety and attentional bias for threat: an event-related potential study. NeuroReport 16, 1501-1505. doi: 10.1097/01.wnr. 0000176522.26971 .83

Liddell, B. J., Williams, L. M., Rathjen, J., Shevrin, H., and Gordon, E. (2004). A temporal dissociation of subliminal versus supraliminal fear perception: an event-related potential study. J. Cogn. Neurosci. 16, 479-486. doi: 10.1162/ 089892904322926809

Luck, S. J. (2014). An Introduction to the Event-Related Potential Technique: Cambridge: MIT press.

Maris, E., and Oostenveld, R. (2007). Nonparametric statistical testing of EEG-and MEG-data. J. Neurosci. Methods 164, 177-190. doi: 10.1016/j.jneumeth.2007.03. 024

Mensen, A., and Khatami, R. (2013). Advanced EEG analysis using threshold-free cluster-enhancement and non-parametric statistics. Neuroimage 67, 111-118. doi: 10.1016/j.neuroimage.2012.10.027

Okruszek, Ł. (2018). It is not just in faces! processing of emotion and intention from biological motion in psychiatric disorders. Front. Hum. Neurosci. 12:48. doi: 10.3389/fnhum.2018.00048

Oostenveld, R., Fries, P., Maris, E., and Schoffelen, J. M. (2011). Fieldtrip: open source software for advanced analysis of MEG, EEG, and invasive electrophysiological data. Comput. Intell. Neurosci. 2011:156869. doi: 10.1155/ 2011/156869

Pichon, S., de Gelder, B., and Grèzes, J. (2012). Threat prompts defensive brain responses independently of attentional control. Cereb. Cortex 22, 274-285. doi: 10.1093/cercor/bhr060

Pierno, A. C., Becchio, C., Turella, L., Tubaldi, F., and Castiello, U. (2008). Observing social interactions: the effect of gaze. Soc. Neurosci. 3, 51-59. doi: $10.1080 / 17470910701563269$

Polich, J. (2007). Updating P300: an integrative theory of P3a and P3b. Clin. Neurophysiol. 118, 2128-2148. doi: 10.1016/j.clinph.2007.04.019

Price, T. F., Dieckman, L. W., and Harmon-Jones, E. (2012). Embodying approach motivation: body posture influences startle eye blink and event-related potential responses to appetitive stimuli. Biol. Psychol. 90, 211-217. doi: 10.1016/j. biopsycho.2012.04.001

Rellecke, J., Sommer, W., and Schacht, A. (2012). Does processing of emotional facial expressions depend on intention? time-resolved evidence from eventrelated brain potentials. Biol. Psychol. 90, 23-32. doi: 10.1016/j.biopsycho.2012. 02.002

Sartori, L., Becchio, C., Bara, B. G., and Castiello, U. (2009). Does the intention to communicate affect action kinematics? Conscious. Cogn. 18, 766-772. doi: 10.1016/j.concog.2009.06.004

Schimmack, U., and Derryberry, D. E. (2005). Attentional interference effects of emotional pictures: threat, negativity, or arousal? Emotion 5, 55-56. doi: 10. 1037/1528-3542.5.1.55

Schupp, H. T., Öhman, A., Junghöfer, M., Weike, A. I., Stockburger, J., and Hamm, A. O. (2004). The facilitated processing of threatening faces: an ERP analysis. Emotion 4, 189-200. doi: 10.1037/1528-3542.4.2.189

Schutter, D. J. L. G., Hofman, D., and Van Honk, J. (2008). Fearful faces selectively increase corticospinal motor tract excitability: a transcranial magnetic stimulation study. Psychophysiology 45, 345-348. doi: 10.1111/j.14698986.2007.00635.x

Steel, K., Ellem, E., and Baxter, D. (2015). The application of biological motion research: biometrics, sport, and the military. Psychon. Bull. rev. 22, 78-87. doi: 10.3758/s13423-014-0659-5

Tamietto, M., and De Gelder, G. B. (2010). Neural bases of the non-conscious perception of emotional signals. Nat. Rev. Neurosci. 11, 697-709. doi: 10.1038/ nrn2889

Thierry, G., Pegna, A. J., Dodds, C., Roberts, M., Basan, S., and Downing, P. (2006). An event-related potential component sensitive to images of the human body. Neuroimage 32, 871-879. doi: 10.1016/j.neuroimage.2006.03.060

Thigpen, N. N., Kappenman, E. S., and Keil, A. (2017). Assessing the internal consistency of the event-related potential: an example analysis. Psychophysiology 54, 123-138. doi: 10.1111/psyp.12629

Thorpe, S., Fize, D., and Marlot, C. (1996). Speed of processing in the human visual system. Nature 381, 520-522. doi: 10.1038/381520a0 
Uusberg, A., Uibo, H., Kreegipuu, K., Tamm, M., Raidvee, A., and Allik, J. (2013). Unintentionality of affective attention across visual processing stages. Front. Psychol. 4:969. doi: 10.3389/fpsyg.2013.00969

van Heijnsbergen, C. C. R. J., Meeren, H. K. M., Grèzes, J., and de Gelder, B. (2007). Rapid detection of fear in body expressions, an ERP study. Brain Res. 1186(Suppl. C), 233-241. doi: 10.1016/j.brainres.2007.09.093

van Loon, A. M., van den Wildenberg, W. P. M., van Stegeren, A. H., Ridderinkhof, K. R., and Hajcak, G. (2010). Emotional stimuli modulate readiness for action: a transcranial magnetic stimulation study. Cogn. Affect. Behav. Neurosci. 10, 174-181. doi: 10.3758/cabn.10.2.174

Weinberg, A., and Hajcak, G. (2010). Beyond good and evil: the time-course of neural activity elicited by specific picture content. Emotion 10, 767-782. doi: $10.1037 / \mathrm{a} 0020242$

Wei, P., Wang, D., and Ji, L. (2016). Reward expectation regulates brain responses to task-relevant and task-irrelevant emotional words: ERP evidence. Soc. Cogn. Affect. Neurosci. 11, 191-203. doi: 10.1093/scan/nsv097

Williams, L. M., Palmer, D., Liddell, B. J., Song, L., and Gordon, E. (2006). The 'when' and 'where' of perceiving signals of threat versus non-threat. Neuroimage 31, 458-467. doi: 10.1016/j.neuroimage.2005.12.009
Yiend, J., and Mathews, A. (2001). Anxiety and attention to threatening pictures. Q. J. Exp. Psychol. 54, 665-681. doi: 10.1080/713755991

Yuan, J., Zhang, Q., Chen, A., Li, H., Wang, Q., Zhuang, Z., et al. (2007). Are we sensitive to valence differences in emotionally negative stimuli? electrophysiological evidence from an ERP study. Neuropsychologia 45, 2764-2771. doi: 10.1016/j.neuropsychologia.2007. 04.018

Conflict of Interest Statement: The authors declare that the research was conducted in the absence of any commercial or financial relationships that could be construed as a potential conflict of interest.

Copyright (C) 2018 Wang, Wang, Luo and Nan. This is an open-access article distributed under the terms of the Creative Commons Attribution License (CC BY). The use, distribution or reproduction in other forums is permitted, provided the original author(s) and the copyright owner(s) are credited and that the original publication in this journal is cited, in accordance with accepted academic practice. No use, distribution or reproduction is permitted which does not comply with these terms. 